\title{
Cerebellum as a Master-Piece for Linguistic Predictability
}

\author{
Peter Mariën $^{1,2}$ • Mario Manto ${ }^{3,4}$
}

Published online: 25 October 2017

(C) Springer Science+Business Media, LLC 2017

During the past decades, a wealth of accumulating evidence from neuroanatomical, neurophysiological, neuroimaging, and clinical studies has substantially altered the traditional view on the cerebellum as a sole coordinator of sensorimotor functions. Studies have identified a non-motor role of the cerebellum and have clearly established that this unique structure is also crucially involved in a higher-level processing including various domains of cognition, language, affect, and behavior [1].

Inspired by the seminal works of Snider (e.g., [2]), Dow (e.g., [3]), Heath (e.g., [4]), and others (see [5] for a review), investigators in the mid-1900s started to examine a possible link between the cerebellum, cognition, and emotion. This laid a foundation for the rediscovery of "cerebellar cognition" by Leiner and colleagues $[6,7]$ who hypothesized that the more recently evolved parts of the cerebellum (neocerebellum/lateral cerebellum connecting with the supratentorial association areas not involved in sensorimotor control) contribute to learning, cognition, and language. Schmahmann introduced the dysmetria of thought hypothesis, providing a historical, clinical, neuroanatomical, and theoretical framework within which a cerebellar role in higher cognitive

Peter Mariën

peter.marien5@telenet.be

1 Clinical and Experimental Neurolinguistic, Vrije Universiteit Brussel, Pleinlaan 2, B-1050 Brussels, Belgium

2 Department of Neurology and Memory Clinic, ZNA Middelheim, Lindendreef 1, B-2020 Antwerp, Belgium

3 Unité d'Étude du Mouvement, FNRS, ULB-Erasme, Route de Lennik 808, B-1070 Brussels, Belgium

4 Service des Neurosciences, UMons, 7000 Mons, Belgium and affective processes could be considered [8,9]. Within these frameworks, Schmahmann and Sherman [9] developed the influential concept cerebellar cognitive affective syndrome (CCAS/Schmahmann's syndrome), the third cornerstone in clinical ataxiology [10].

Schmahmann's syndrome/CCAS consists of a cluster of cognitive and affective symptoms, classified in four categories: (1) executive dysfunctions (planning, set-shifting, abstract reasoning, and working memory), (2) visuospatial deficits (visuospatial organization and memory), (3) behavioral-affective disturbances (blunting of affect or disinhibited and inappropriate behavior), and (4) language symptoms (agrammatism and anomia). Over the past years, an enormous growth of knowledge has been reached in the contribution of the cerebellum to non-motor linguistic processing based on countless neuroimaging and clinical studies addressing the role of the cerebellum in various language domains. Clinical and functional neuroimaging studies advanced the notion of a lateralized (right) posterior cerebellar involvement in non-motor language tasks (lobule VI, Crus I/II, and midline lobule VII of the - right - cerebellum) subserved by crossed, reciprocal cerebello-cerebral loops with the language dominant hemisphere [11-13]. Topographical findings are complemented by clinical studies demonstrating the modulatory role of the postero-lateral (right) cerebellum in a variety of non-motor language deficits. For instance, cerebellar pathology may disrupt several domains of speech/ language perception. Phonological and graphomotor planning and execution deficits of skilled movements may give rise to apraxia of speech or apraxic agraphia. Specific damage of phonemically related retrieval strategies in cerebellar patients are frequently observed and linked to impaired verbal fluency (letter more than word fluency) and adynamic output. Expressive and receptive agrammatism has been reported in various cerebellar 
ataxias. Impaired cerebellar function may lead to reading and writing difficulties as well (for a review, see [12]).

Due to its striking homogeneous cytoarchitecture, it has been assumed that the cerebellar circuitry is an anatomical substratum for adaptive behavior in the motor as well as non-motor domain in a similar way. As a result, important insights about the contribution of the cerebellum to neurocognition might be derived from its involvement in motor control. The notion that the cerebellum encodes internal models (corresponding to any neural representation of the external world, enabling predictions and consequently optimizing motor behavior but also mental representations [14]) is an important recent theoretical development in cognitive neurosciences. Schmahmann referred to this unique computation as the universal cerebellar transform (UCT) $[15,16]$. This mechanism allows the cerebellum to compare predictions of internal models with the incoming feedback from the cerebrum, improving human behavior and performance. This is in accordance with the concept of dysmetria of thought which implies a universal cerebellar impairment $[9,15]$. If the cerebellum is involved in every cortical process in the same way, cerebellar damage should result in a similar kind of disruption of these processes [16]. In this context, every deficit resulting from cerebellar damage could be interpreted as hypometric/ diminished or hypermetric/exaggerated behavior (for an overview, see [16]).

Internal models have been used to explain the ability to perform controlled and skillful movements by creating a prediction of the dynamics of a body part. These predictions help to perform a complex movement in a very precise way without constantly relying on sensory feedback [14]. Ito hypothesized that these internal models can be used in the same manner to create and manipulate mental representations in association with the prefrontal and the temporo-parietal cortex [14]. Via a dense network of cerebro-ponto-cerebello-cerebral pathways, the internal models stored in the cerebellum receive a copy in particular from the fronto-temporal input analysis system processed at each stage. Language comprehension is an example of simultaneous predictions at various linguistic steps, with predictions being produced by the language production system along with the operations performed on it [17].

In language studies, the cerebellum is now regarded as a predictor of future states. Predictions would optimize language functioning by comparing them with the cortical feedback [12]. Within this computational framework, Ito and Argyropoulos, for instance, further extended the contributions of cerebellar internal models to non-motor aspects of predictive language processing [14, 18, 19]. Argyropoulos stated that associative memory traces of contiguous linguistic events are stored in the cerebellum (such as the processing of the phonological, semantic, and syntactic properties of words that will be used) [19]. Internal models would then convey a prediction, via the ventrolateral portion of the dentate nucleus, the parvocellular red nucleus, and the thalamus to the prefrontal cortex. Any incompatibility would elicit error signals back to the cerebellar cortex via the climbing fibers originating from the inferior olive and projecting to both cerebellar nuclei and cerebellar cortex. It has been argued that, on the basis of long-term depression (LTD) of parallel fiber-to-Purkinje cell synapses, these errors train the internal model for accurate predictions [19]. Based on recent fMRI evidence, it is proposed that that lobule HVIIa of the right cerebellum may fulfill this role [19]. However, other cerebellar regions might as well be implicated in different levels of linguistic predictions but this issue remains to be elucidated.

A number of studies provide further evidence for cerebellar internal models transmitting predictions on upcoming "non-motor" information in various linguistic processes including phonology, semantics, language dynamics, reading, writing, and syntax. Lesage et al. conducted an event-related fMRI study in which the predictability of written sentences was manipulated and additionally conducted a semantic, phonological, and orthographic fMRI processing task [20]. Predictability and prediction violation of the target word correlated with the activity in the right posterolateral cerebellum. In addition, involvement of the right postero-lateral cerebellum was observed in phonological, but not in semantic or orthographic processing. Studies applying other methodologies such as rTMS and tDCS also add evidence that the cerebellum fulfills a unifying role in predictability both within the motor and non-motor domain [21-23]. Due to its huge connectivity with the spinal cord, the brainstem and the supra-tentorial structures (including the recently discovered loops between the cerebellum and basal ganglia [24]), the cerebellum appears as a master-piece for predictions in language. Cerebellum contains the highest densities of neurons and is organized into multiple modules having a very high degree of adaptability in terms of synaptic plasticity, two features which render this structure an ideal candidate for an internal machine-learning device. One can anticipate that the secrets underlying the predictions in language will be intensely studied over the next years, given the unique importance of language in advanced cognition and behavior, and the frequent disabling course of ataxic dysarthria [1,25]. Elucidating these secrets will provide key information on how the brain has developed neurobiological strategies to distinguish external sensory inputs from those that are self-generated [26]. So far, computational studies, neuronal recordings, and clinical studies have all pointed to the cerebellar cortex as a key site in which the forward models are built and stored [27-29]. The respective contributions of the cerebellar cortex, cerebellar nuclei, mossy fibers, and climbing 
fibers are 4 major open questions for the understanding of predictions of language in human. This is also a door to dissect the mechanisms and rules of both the evolution of the nervous system and the lateralization processes [30, 31].

Contributions The authors contributed equally to the work.

Funding Information MM is supported by the FNRS and the Fonds Erasme.

\section{Compliance with Ethical Standards}

Conflicts of Interest The authors declare that they have no conflict of interest.

\section{References}

1. Manto M, Gruol DL, Schmahmann JD, Koibuchi N, Rossi F. Handbook of the cerebellum and cerebellar disorders. Dordrecht: Springer; 2013.

2. Snider RS, Eldred E. Cerebral projections to the tactile, auditory and visual areas of the cerebellum. Anat Rec. 1948;100:714.

3. Dow RS. Some novel concepts of cerebellar physiology. Mt Sinai J Med. 1974;41:103-19.

4. Heath RG. Modulation of emotion with a brain pacemaker. Treatment for intractable psychiatric illness. J Nerv Ment Dis. 1977;165:300-17.

5. Schmahmann JD. Rediscovery of an early concept. In: Schmahmann JD, editor. The cerebellum and cognition. Int Rev Neurobiol, vol. 41. San Diego: Academic Press; 1997. p. 3-27.

6. Leiner HC, Leiner AL, Dow RS. Does the cerebellum contribute to mental skills? Behav Neurosci. 1986;100:443-54.

7. Leiner HC, Leiner AL, Dow RS. The human cerebro-cerebellar system: its computing, cognitive, and language skills. Behav Brain Res. 1991;44:113-28.

8. Schmahmann J. An emerging concept. The cerebellar contribution to higher function. Arch Neurol. 1991;48:1178-87.

9. Schmahmann JD, Sherman JC. The cerebellar cognitive affective syndrome. Brain. 1998;121:561-79.

10. Manto M, Mariën P. Schmahmann's syndrome-identification of the third cornerstone of clinical ataxiology. Cerebellum Ataxias. 2015;2(1):2. https://doi.org/10.1186/s40673-015-0023-1.

11. Stoodley CJ, Schmahmann JD. Functional topography in the human cerebellum: a meta-analysis of neuroimaging studies. NeuroImage. 2009;44:489-501.

12. Mariën P, Ackermann H, Adamaszek M, Barwood CHS, Beaton A, Desmond J, et al. Consensus paper: language and the cerebellum: an ongoing enigma. Cerebellum. 2014;13(3):386-410.

13. Mariën P, Manto M. The linguistic cerebellum. London: Academic Press/Elsevier; 2016. 420 pp.
14. Ito M. Control of mental activities by internal models in the cerebellum. Nat Rev Neurosci. 2008;9:304-13.

15. Guell X, Gabrieli JDE, Schmahmann JD. Embodied cognition and the cerebellum: perspectives from the dysmetria of thought and the universal cerebellar transform theories. Cortex. 2017; https://doi. org/10.1016/j.cortex.2017.07.005.

16. Koziol LF, Budding D, Andreasen N, D'Arrigo S, Bulgheroni S, Imamizu $\mathrm{H}$, et al. Consensus paper: the cerebellum's role in movement and cognition. Cerebellum. 2014;3(1):151-77.

17. Pickering MJ, Garrod S. Do people use language production to make predictions during comprehension? Trends Cogn Sci. 2007;11(3):105-10.

18. Ito M. Neural control of cognition and language. In: Marantz A, Miyashita Y, O’Neil W, editors. Image, language, brain. Cambridge: MIT Press; 2000. p. 149-62.

19. Argyropoulos GP. The cerebellum, internal models and prediction in "non-motor" aspects of language: a critical review. Brain Lang. 2016;161:4-17.

20. Lesage E, Hansen PC, Miall RC. Right lateral cerebellum represents linguistic predictability. J Neurosci. 2017;37(26):6231-41.

21. Runnqvist E, Bonnard M, Gauvin HS, Attarian S, Trebuchon A, Hartsuiker RJ, et al. Internal modeling of upcoming speech: a causal role of the right posterior cerebellum in non-motor aspects of language production. Cortex. 2016;81:203-14.

22. Miall RC, Antony J, Goldsmith-Sumner A, Harding SR, McGovern C, Winter JL. Modulation of linguistic prediction by TDCS of the right lateral cerebellum. Neuropsychologia. 2016;86:103-9.

23. D'Mello AM, Turkeltaub PE, Stoodley CJ. Cerebellar tDCS modulates neural circuits during semantic prediction: a combined tDCSfMRI study. J Neurosci. 2017;37:1604-13.

24. Caligiore D, Pezzulo G, Baldassarre G, Bostan AC, Strick PL, Doya $\mathrm{K}$, et al. Consensus paper: towards a systems-level view of cerebellar function: the interplay between cerebellum, basal ganglia, and cortex. Cerebellum. 2017;16(1):203-29.

25. Mansouri FA, Koechlin E, Rosa MGP, Buckley MJ. Managing competing goals - a key role for the frontopolar cortex. Nat Rev Neurosci. 2017; https://doi.org/10.1038/nrn.2017.111.

26. Sawtell NB. Neural mechanisms for predicting the sensory consequences of behavior: insights from electrosensory systems. Annu Rev Physiol. 2017;79:381-99.

27. Ebner TJ, Pasalar S. Cerebellum predicts the future motor state. Cerebellum. 2008;7(4):583-8.

28. Herzfeld DJ, Kojima Y, Soetedjo R, Shadmehr R. Encoding of action by the Purkinje cells of the cerebellum. Nature. 2015;526(7573):439-42.

29. Blazquez PM, Kim G, Yakusheva TA. Searching for an internal representation of stimulus kinematics in the response of ventral paraflocculus Purkinje cells. Cerebellum. 2017;16(4):817-26.

30. Sousa AMM, Meyer KA, Santpere G, Gulden FO, Sestan N. Evolution of the human nervous system function, structure, and development. Cell. 2017;170(2):226-47.

31. Corballis MC. The evolution of lateralized brain circuits. Front Psychol. 2017;8:1021. https://doi.org/10.3389/fpsyg.2017.01021. eCollection 2017 